\title{
Where in the Virgo Cluster are Galaxies Stripped? Stellar Population Evolution of Stripped Spiral Galaxies in Virgo
}

\author{
Hugh H. Crowl ${ }^{1}$ and Jeffrey D. P. Kenney ${ }^{1}$ \\ ${ }^{1}$ Department of Astronomy, Yale University, P.O. Box 208101, New Haven, CT, 06520, USA \\ email: hugh@astro.yale.edu
}

\begin{abstract}
The nearby Virgo Cluster provides an ideal laboratory to study galaxy-galaxy and galaxy-cluster interactions at a level of detail impossible at higher redshift. In Virgo, there exists a large population of spiral galaxies with mostly undisturbed stellar disks, but truncated gas disks. We present results of an observational study of several of these galaxies, utilizing optical and UV imaging, and optical spectroscopy. By combining optical spectroscopy and UV imaging, we are able to constrain the time since star formation ended in the outer disk and, therefore, constrain the time since the galaxies were stripped. Our results show that while most of the galaxies in our sample are consistent with being stripped near the cluster center, several show evidence for being stripped well outside the core, suggesting that the "reach" of the intracluster medium is greater than is suggested by simple ICM models.
\end{abstract}

\section{Introduction}

The morphology-density relationship (Dressler 1980) implies that clusters are creating S0 galaxies at the expense of spirals. The most salient difference between the two galaxy populations is the presence (spirals) or absence (S0s) of current star formation. Kauffmann et al.(2004) find that the star formation - environment correlation is the strongest correlation of any galaxy property with environment, suggesting that environment plays a key role in the termination of star formation. While there is intense debate as to the specific environmental processes that cause the transformation, stellar population simulations (Shioya et al. 2004) have shown that simple truncation of star formation in a spiral galaxy can form S0-type spectra.

In the nearby Virgo cluster, there exists a population of spiral galaxies with normal star formation inside some radius, but little or no star formation beyond that "truncation radius" (Koopmann \& Kenney 2004). Many of these galaxies have relatively undisturbed stellar distributions, despite their severely truncated $\mathrm{H} \alpha$ disks. Such a disturbance in the gas with little or no apparent stellar disk disturbance strongly suggests a gas-gas interaction; if a gravitational interaction disturbed the star formation, the stellar light should also be disturbed. The appearance of these galaxies beyond the stripping radius (i.e. normal stellar disk with no current star formation) is strikingly similar to the passive spirals in higher redshift clusters. By studying the stellar populations of our sample galaxies through optical spectroscopy and UV imaging, we aim to understand when and where in the cluster galaxies are stripped. The location of stripping tells us how effective stripping is at different distances from the center of the cluster. Simple models of ISMICM stripping suggest that stripping can only occur in the cluster core, where the ICM densities are highest. Galaxies stripped far from the center of the cluster suggest a nonstatic ICM or extreme conditions outside the cluster core. 


\section{Results}

If it is the case that galaxies are only (or primarily) stripped in the core, this implies that there should be a relationship between galaxies' location in the cluster and time since star formation truncation. While projection effects limit our understanding of the true distance between a galaxy and M87 (at the core of Virgo), we can set lower limits to the clustercentric distance by using the projected distance from M87 $\left(d_{87}\right)$.

In Figure 1, we show the distance of the galaxies from M87 plotted against the time since star formation ended $\left(t_{\mathrm{tr}}\right)$ for each galaxy. The dashed line in Figure 1 shows the distance a galaxy could travel in a given

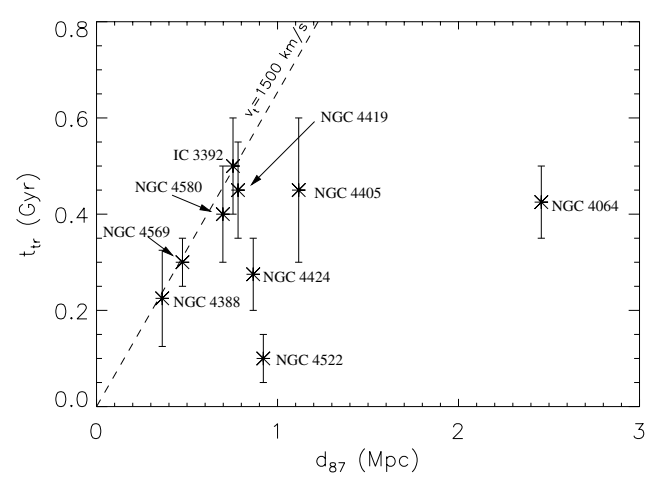

Figure 1. Time since the truncation of outer disk star formation plotted against the projected distance from the giant Elliptical at the center of Virgo: M87. time if its velocity was $1500 \mathrm{~km} / \mathrm{s}$ in the plane of the sky. The average line-of-sight velocity of a galaxy in Virgo relative to the cluster is $\sim 700 \mathrm{~km} / \mathrm{s}$, so $1500 \mathrm{~km} / \mathrm{s}$ is a conservative estimate for most galaxies. Any galaxies to the right of the line in the figure are too far out to have been stripped in the cluster core. While most galaxies are consistent with being stripped in the core, there are two galaxies clearly to the right of the line.

Despite the distance from the cluster core, the stellar population of NGC 4064 points to star formation truncation only $425 \mathrm{Myr}$ ago. It is possible that this galaxy was stripped of it's star forming gas by an interaction with the ICM, but if that is the case, it did not happen in the core of Virgo. Optical photometry, spectroscopy, and radio molecular gas interferometry (Cortés et al. 2006) suggest that this galaxy has recently undergone a minor gravitational interaction. The HI is truncated well within the stellar disk of NGC 4064, demonstrating that the gas was removed without significantly disrupting the stars. This galaxy has clearly lost a large fraction of its gas, but it's not clear if simple ISM-ICM stripping alone could cause the observed state. However, it is apparent that significant gas has been stripped from this galaxy far removed from the Virgo core. NGC 4522, despite being closer to the cluster center than NGC 4064, has an exceedingly young population $\left(t_{\mathrm{tr}} \approx 100 \mathrm{Myr}\right)$ that precludes it from being stripped in the core. Simple estimates of ram pressure are too low by a factor of 10 to adequately explain the observed stripping. However, the estimates of ram pressure in assume a static ICM; the ICM is clearly dynamic and observed ICM shocks (Shibata et al. 2001) suggest that the ICM may be moving with significant velocity in the region of NGC 4522. Taken together these results suggest that, while many galaxies are consistent with being stripped in the core of Virgo, the "reach" of the ICM is greater than simple estimates imply and can affect galaxies outside the cluster core.

\section{References}

Cortés, J. R., Kenney, J. D. P., \& Hardy, E. 2006, AJ, 131, 747

Dressler, A. 1980, ApJ, 236, 351

Kauffmann, G. et al. 2004, MNRAS, 353, 713

Koopmann, R. A., \& Kenney, J. D. P. 2004, ApJ, 613, 866

Shibata, R. et al. 2001, ApJ, 549, 228

Shioya, Y., Bekki, K., \& Couch, W. J. 2004, ApJ, 601, 654 